\title{
La autonomización de hospitales públicos y sus consecuencias sobre la demanda de atención de salud, Lima, 1988-1997
}

\author{
Juan Arroyo Laguna ${ }^{1}$
}

RESUMEN Entre 1988 y 1992 los hospitales públicos del Perú fueron afectados por grandes cambios: el colapso operativo de los servicios de salud, una profunda crisis presupuestaria del Estado y una serie de modificaciones tendentes al autofinanciamiento, cambios anteriores a las leyes de reforma sanitaria de 1997. En los últimos años, esos cambios en la política de financiamiento han redundado en la renovación de la potencialidad operativa de todo el sector de la salud y de la actividad hospitalaria. Por otra parte, ha habido indicios de que la generación de recursos propios en los hospitales mediante el cobro de tarifas ha modificado la proporción de usuarios de distintos estratos socioeconómicos en desmedro de los más pobres. Se planteó, entonces, la posibilidad de que la generación de recursos propios fuera inconveniente para la población necesitada de servicios gratuitos. En el estudio, por lo tanto, se procuró descubrir si habían cambiado los estratos socioeconómicos de los usuarios que acudieron entre los años 1988 y 1997 a cinco grandes hospitales públicos de Lima y la asociación que pudiera haber con la actual transición hacia el nuevo modelo de hospital autónomo, en particular con la nueva política de financiamiento hospitalario.

Los cambios en la oferta hospitalaria se estudiaron mediante entrevistas en profundidad de informantes clave, la recopilación de datos estadísticos en los establecimientos seleccionados y una vasta revisión bibliográfica y documentaria. Los cambios en la demanda hospitalaria se estudiaron aplicando 1012 encuestas a usuarios hospitalarios de los años 1988, 1992 y 1997. Para clasificar por estrato socioeconómico a esos usuarios, se utilizaron el análisis de conglomerados y el análisis discriminante, basándose en seis variables apropiadas para establecer esos niveles. El modelo resultante, de tres grupos socioeconómicos según las variables dicotomizadas, superó los 75 puntos exigidos en la prueba de clasificación con el análisis discriminante.

Los resultados centrales mostraron que, entre los usuarios de los cinco establecimientos de Lima, el estrato de mayores recursos había aumentado su presencia relativa de 35,4\% en 1988 a 52,6\% en 1997, mientras que el estrato de menores recursos la había disminuido en el mismo período, de 25,4\% a 20,5\%. El estrato intermedio también disminuyó, de 39,2\% a 26,8\%. Puede afirmarse que la tendencia en el financiamiento de los hospitales ha sido el incremento de los copagos entre el estado y la población. El tesoro público pesa relativamente menos en los presupuestos de los hospitales. Como resultado, tal como demostraron las Encuestas Nacionales de Niveles de Vida de 1991 y 1994, la demanda de servicios hospitalarios por personas pobres disminuyó de 37,3 a 34\% entre 1991 y 1994, mientras que en ese mismo período la demanda de personas no pobres aumentó de 34,8 a 43,8\%. Se concluye que las microrreformas sin un proyecto holístico de reforma producen microeficiencia e inequidad sistémica a la vez. En ese contexto, la producción y el rendimiento llegan a cobrar mayor importancia que el impacto y la efectividad de los servicios en la población servida.

1 Dirección postal: Universidad Peruana Cayetano Heredia, Facultad de Salud Pública y Administración, Av. Honorio Delgado 430, Lima 31, Lima, Perú, tef. (511) 4820302, fax (511) 3819072, jarroyo@upch.edu.pe
El comienzo de la actual reforma de los hospitales en el Perú puede ubicarse entre 1988 y 1991, mucho antes de que aparecieran, a partir de mayo de 1997, los primeros dispositivos legales de la reforma sectorial (Ley 
26790 de Modernización de la Seguridad Social). Fue, por lo tanto, muy anterior al intento de una reforma general explícita y puede considerarse un caso paradigmático de reforma sanitaria silenciosa, es decir, de una transformación ad hoc que ocurrió sin que existiera un diseño sistémico ni jurídico y se realizó por acumulación de reformas parciales. En el período de 1988 a 1997 los hospitales públicos del Perú fueron afectados por una evolución del sector de la salud, que atravesó por dos etapas. La primera se desarrolló entre 1988 y 1992, y se caracterizó por el denominado colapso operativo de los servicios, la profunda crisis presupuestaria del Estado y las consiguientes modificaciones hacia el autofinanciamiento de los establecimientos públicos de salud. En una segunda etapa que empezó en 1993 y llega hasta la actualidad, se ha renovado la potencialidad operativa de todo el sector de la salud y, en particular, la actividad hospitalaria.

A principios de los años noventa se inició un cambio de financiamiento hospitalario que obedeció a una razón muy práctica. El Perú estaba en medio de una aguda crisis fiscal y los servicios públicos habían llegado a una situación que el propio MINSA caracterizaba como colapso operativo (1). La idea original fue financiar con recursos autogenerados los servicios destinados a los usuarios exonerados de tarifas en razón de su extrema pobreza. De esta forma, los grupos de la población que, por no ser pobres, estaban usando el subsidio público indebidamente financiarían mediante el pago de tarifas a los grupos más necesitados. De hecho, en cada hospital funciona un departamento de servicio social, donde los usuarios que no pueden pagar la tarifas acuden para recibir una calificación social. Esa calificación ha sido muy inexacta pues se determina de forma poco profesional. Además, no existen estudios sobre el número de pacientes atendidos en cada establecimiento que señalen los porcentajes de pobres y no pobres entre sus usuarios y que sirvan para que cada hospital fije cuotas de beneficiarios y prestaciones gratuitas. El MINSA tampoco asigna esas cuotas desde las direc- ciones regionales de salud respectivas. Como resultado de todo ello, en los hospitales públicos se han tenido que tomar decisiones que corresponden a las instancias gubernamentales del sector de la salud y, por medio de esas decisiones cotidianas, elaborar una política hospitalaria. En estos casos, las transformaciones han derivado principalmente de la iniciativa de los actores sociales del sector de la salud, en particular, de la tecnocracia emergente y los directores institucionales.

En el caso del Perú, la autonomización de los hospitales emanó principalmente de un cambio en la fuente de financiación, el cual se ha reflejado progresivamente en márgenes más amplios de autonomía y un nuevo estilo de gestión. Una vez que los hospitales públicos comenzaron a generar recursos propios mediante el cobro de tarifas a ciertos usuarios, lograron ciertos márgenes de flexibilidad para utilizar esos ingresos, lo que se convirtió con el tiempo en un incentivo para redirigir sus actividades hacia el incremento de ingresos variables mediante el cobro de copagos. La autonomización hospitalaria en el Perú no significa una desvinculación con el Ministerio de Salud sino una flexibilización para autogenerar sus recursos y usufructuarlos. Dado que en el sistema de atención peruano las asignaciones de niveles de atención a los hospitales no les impiden atender consultas de primer nivel, los hospitales en la práctica compiten con los centros de salud y las postas sanitarias. Los centros y postas tienen en común el hecho de que brindan servicios básicos pero difieren en el nivel de complejidad y la capacidad de resolución. Desde antes de 1990 era innecesario que los pacientes acudieran al nivel de atención primario para obtener acceso a la atención hospitalaria. En función de captar mayores recursos, los hospitales públicos han utilizado esta posibilidad de expansión en todos los niveles de atención.

Ese cambio en el financiamiento hospitalario iniciado alrededor de 1990 ha modelado, entonces, un tipo de autonomización hospitalaria semiespontánea, diferente de la autonomización sistémica que se debate en la literatura sobre reformas en el campo de la salud (2-5). Como resultado, las unidades de servicio se han vuelto más productivas y relativamente más modernas. Se ha dado una transición de facto del hospital estatal público de los años setenta y ochenta a un nuevo hospital de financiamiento mixto y régimen semiautónomo, transición que, según todos los indicios observables, implicaría un cambio en el público tradicional objetivo de la atención de salud hospitalaria. Considerando la importancia de evaluar las reformas de salud no solo desde el punto de vista de los servicios sino desde el de la población, debe estarse atentos a las consecuencias imprevistas de las diversas transiciones descritas. Sobre todo, es preciso resolver si una modernización hospitalaria de este tipo puede estar al servicio de una ética pública o no.

Se postuló, en consecuencia, la hipótesis de que esos cambios podrían tener consecuencias imprevistas en la demanda de atención, lo que impulsó a estudiar y esclarecer la situación. El objetivo principal de la investigación fue averiguar si, junto con el proceso de autonomización de los hospitales, se había registrado alguna transformación en los grupos de usuarios que acudían a los hospitales. Sin embargo, la selección para el estudio de cinco de los más importantes hospitales públicos de Lima permitió ir más allá de cinco estudios de casos y captar las características generales del proceso de reforma silenciosa de los hospitales del Estado en los años noventa y su impacto sobre la demanda de atención.

Si bien la investigación se centró en gran medida en los usuarios atendidos en cinco hospitales públicos de Lima, Perú, entre 1988 y 1997, el estudio comprendió también una indagación de las modificaciones de la oferta en ese período, en particular los cambios en el financiamiento de la atención hospitalaria. Reconstruyendo la información sobre consultas externas se buscó conocer también si los cambios en las fuentes de financiamiento habían sido acompañados de modificaciones en la producción de esos servicios. El interés principal de este estudio reside en documentar información que permita 
compatibilizar la búsqueda de mayor eficiencia en el uso de los recursos con la necesidad de mejor acceso y orientación del subsidio público hacia los más necesitados.

\section{MATERIALES Y MÉTODOS}

Este estudio se llevó a cabo durante el período de mayo de 1996 a febrero de 1997. En 1996, el MINSA administraba en Lima 22 establecimientos hospitalarios de diferente nivel; en realidad siete institutos especializados y 15 hospitales, con un total de 6505 camas (6). Para el estudio se seleccionaron cinco nosocomios del área metropolitana de Lima, uno por cada una de las subregiones administrativas de salud en las que se divide la capital del Perú: Daniel Alcides Carrión, Cayetano Heredia, Arzobispo Loayza, María Auxiliadora e Hipólito Unanue. Los cinco hospitales tenían 3394 camas en conjunto, es decir, 52,2\% de las camas hospitalarias administradas por el MINSA en Lima. Se trata de hospitales del más alto nivel dentro del sistema de atención peruano; cuatro de ellos tienen el rango de hospitales de referencia nacionales y uno, de referencia regional. Sin embargo, la diferencia formal no significa una diferencia en el nivel de atención que ofrecen, debido a que los cinco establecimientos tienen características tecnológicas similares.

La complejidad del objeto de estudio - un proceso social que involucra políticas institucionales y mercadeo de servicios de salud - requirió el empleo de una metodología interdisciplinaria con enfoques y técnicas provenientes de diferentes campos. La transformación que ocurrió de 1988 a 1997 en los cinco hospitales públicos seleccionados se estudió mediante entrevistas con informantes clave, que fueron los directores y exdirectores de los hospitales, funcionarios de sus equipos de gestión y autoridades del Ministerio; recopilación de estadísticas de fuentes secundarias y una revisión exhaustiva de información bibliográfica y documental. Para las entrevistas se confeccionó un directorio de informantes clave y un cuestionario semiestructurado con preguntas sobre los cinco hospitales en la etapa de 1988 a 1997. La recopilación estadística, centrada en las fuentes de financiamiento de los hospitales y la producción de consultas, se hizo en las oficinas de planificación, estadística y contabilidad de los hospitales, donde la información desagregada se seleccionó, consolidó y tabuló. La revisión bibliográfica y documentaria se llevó a cabo en instituciones y bibliotecas particulares, mediante el compendio de materiales que permitieron reconstruir el proceso histórico de los hospitales.

Por otra parte, la evolución de los grupos de usuarios se estudió por medio de una encuesta de una muestra de los pacientes que acudieron a los hospitales durante 1988, 1992 y 1997. En la encuesta se consideró como universo la población total de pacientes ambulatorios de los cinco establecimientos en los tres años mencionados $\mathrm{y}$, como unidad de análisis, al paciente hospitalario de consulta externa. Para comparar entre sí a los pacientes que acudieron a los consultorios de los nosocomios seleccionados en los tres años mencionados, se diseñaron tres muestras, cada una representativa de su cohorte de usuarios. Dado que la suma de los pacientes de los cinco hospitales en cualquiera de los tres años superaba el parámetro estadístico de 100 000, el tamaño de las muestras se calculó usando la fórmula de muestras para universos infinitos $n=\lambda p q / E^{2}$. Se calculó un error de $3 \%$, con $\lambda=2(95 \%)$ y $p$ como 19,5 , sobre la base de la proporción promedio de indigentes o pobres extremos en los 10 años anteriores. Se obtuvo así la cifra muestral de 350 pacientes para cada año. Seguidamente se confeccionaron listas de pacientes de 1988 y 1992 para entrevistar, seleccionando de forma aleatoria historias clínicas de dichos años entre los archivos hospitalarios. Cada hospital aportó a la muestra un número de pacientes proporcional al número atendido en la consulta externa en los años considerados. Los pacientes fueron visitados en sus domicilios. Los 350 pacientes de 1997 fueron seleccionados y entrevistados aleatoriamente en las salas de espera de los consultorios externos de los cinco hospitales.
En las encuestas se utilizó un cuestionario estructurado de 89 preguntas con secciones sobre familia, salud, vivienda, educación, transporte, empleo e ingresos y gastos. Antes del trabajo de campo se realizó una encuesta piloto, que permitió corregir y validar el cuestionario utilizado.

Se logró entrevistar a 1012 pacientes de los 1050 de la muestra, esto es, a $96,3 \%$ de los pacientes seleccionados. Las encuestas estuvieron a cargo de un equipo de encuestadores y supervisores que cubrieron la muestra por distritos, de forma que la realización de las entrevistas fue supervisada durante el propio trabajo de campo y el control de calidad pudo aplicarse simultáneamente a la administración de las encuestas.

Los datos obtenidos se registraron en tres bases de datos Fox Pro, una por cada año de estudio, para luego proceder al análisis estadístico y la interpretación. Los grupos de usuarios se clasificaron por estrato socioeconómico utilizando el análisis de conglomerados y el análisis discriminante. Como se sabe, el primero constituye una técnica de carácter clasificatorio y el segundo, además, un recurso estadístico capaz de confirmar o rechazar los agrupamientos brindados por el primero (7).

Para el análisis de conglomerados, se seleccionaron seis variables capaces de discriminar entre los niveles socioeconómicos de los usuarios, conforme a los estudios nacionales realizados en el Perú por el Instituto Nacional de Estadística e Informática, Cuanto, S.A. y Apoyo Opinión y Mercado, S.A. (8-10), las tres entidades encuestadoras de mayor prestigio en el país. Esas variables fueron: nivel de instrucción, grado de hacinamiento, tenencia de la vivienda, situación laboral, autopercepción social y dependencia intrafamiliar (o proporción de personas con y sin empleo en la familia). Dichas variables se consideraron de forma dicotómica para clasificar la muestra de pacientes en tres subgrupos socioeconómicos. El modelo resultante fue luego sometido a prueba mediante el análisis discriminante.

Como está establecido, el análisis discriminante ratifica los agrupamientos clasificatorios surgidos del análisis 
de conglomerados si se logra superar los 75 puntos en la prueba de clasificación. El modelo construido tuvo 80,55 puntos. El análisis discriminante permitió, además, confirmar el ordenamiento preciso de los conglomerados en orden descendente, de forma que el subgrupo 1 quedó establecido como el segmento social relativamente más pudiente y el 3 como el relativamente menos pudiente, con el subgrupo 2 como segmento socioeconómico intermedio. Además, la prueba lambda de Wilks reconfirmó los resultados del análisis de conglomerados con un valor $P$ estadísticamente significativo $(P<0,01)$.

Posteriormente, el análisis discriminante se volvió a utilizar como instrumento para detectar la evolución de los subgrupos de usuarios, ya no por año sino por hospital, con objeto de determinar si en los cinco hospitales se había observado la tendencia general de alejamiento de los conglomerados menos pudientes o si alguno había tenido experiencias diferentes.

\section{RESULTADOS}

La investigación reveló que desde 1988 hasta 1997 el Perú fue el escenario de una reforma tendente a reemplazar el modelo clásico de hospital estatal por un nuevo modelo de hospital autónomo. Especialmente en cuanto a organización y gestión de los servicios, en los años noventa ha desaparecido paulatinamente el modelo heredado de la anterior reforma desarrollada durante los decenios de 1960 y 1970, fase de apogeo de la atención estatal de salud. La configuración hospitalaria estatal clásica se resumía en la prestación con internación, dependencia del Estado, atención gratuita en el momento de la prestación y régimen de trabajo a sueldo del personal (11). Sin embargo, los hospitales públicos limeños que hemos estudiado tienden a definirse cada vez menos por esas características. Conservan algo de todo ello pero ya no son los tradicionales hospitales del Estado de hace 20 años. Hoy en día, sus componentes de hostelería e internación son variables, el financiamiento es tanto público como privado, la atención se rige por tarifas categorizadas y además del personal de planilla, hay cada vez más personal contratado por servicios especiales o por producto así como empleados temporales. Si bien pueden llamarse hospitales públicos estatales por su origen, ya no lo son estrictamente por sus características actuales. En el momento de finalizar este estudio, en los cinco hospitales era notable la tendencia a reformular su relación con el Estado y cobrar mayor autonomía, sobre todo en el planeamiento y la gestión de sus recursos. No obstante, esta tendencia de las entidades públicas de servicios no es sinónimo de privatización, según el MINSA (12).

En el período de estudio, se produjeron cuatro grandes modificaciones en los hospitales públicos estudiados: 1) el cambio hacia un régimen de autodeterminación hospitalaria supervisada por el MINSA; 2) la ejecución de una nueva política de financiamiento; 3) el paso del colapso operativo de principios de los años noventa a una fuerte reexpansión de la producción de servicios; y 4) la modernización de la gestión y la tecnología hospitalarias. Estas cuatro modificaciones han sido promovidas por un proceso de autonomización que se ha generado de forma semiespontánea y dicho proceso ha repercutido en un cambio de los estratos socioeconómicos entre los usuarios tradicionalmente servidos en los cinco hospitales públicos seleccionados. El cuadro 1 muestra la matriz clasificatoria de los conglomerados de usuarios según las variables empleadas.
En lo que se refiere a la demanda hospitalaria atendida, en el período de 1988 a 1997 se produjo un desplazamiento de los segmentos socioeconómicos que tradicionalmente utilizaban los servicios de salud hospitalarios. Los datos obtenidos sobre los tres conglomerados o subgrupos que integraron la muestra de población indicaron el crecimiento del más pudiente (subgrupo 1) y el decrecimiento de los dos menos pudientes (subgrupos 2 y 3) durante los 10 años considerados (figura 1). Puede observarse que ese cambio en la composición de la demanda hospitalaria redujo de forma importante la presencia del subgrupo 2, que bajó 13 puntos en relación al subgrupo 1, mientras que el subgrupo 3 cedió 5 puntos al subgrupo 1. El número de usuarios del subgrupo 1, el relativamente más pudiente, creció notablemente, de 35,5 a $52,6 \%$, mientras que el del subgrupo 2 , de ingresos intermedios, disminuyó de 39,2 a $26,8 \%$ y el del subgrupo 3 , el relativamente menos pudiente, decreció de 25,4 a $20,5 \%$.

El análisis por hospital de la evolución de la demanda mostró que cuatro de los cinco hospitales siguieron en mayor o menor medida la tendencia general de crecimiento del subgrupo 1 y decrecimiento de los subgrupos 2 y 3 . En unos casos más que en otros, los pacientes del estrato de relativamente mayores recursos aumentaron su presencia en los hospitales Daniel A. Carrión, Cayetano Heredia, Arzobispo Loayza y María Auxiliadora. Por el contrario, el hospital Hipólito Unanue siguió un patrón inverso: el subgrupo 1 disminuyó

CUADRO 1. Matriz de clasificación socioeconómica de muestras de usuarios de cinco hospitales públicos, Lima, Perú, 1988, 1992 y 1997

\begin{tabular}{lcccccc}
\hline \multicolumn{1}{c}{ Subgrupo } & $\begin{array}{c}\text { Tipo de } \\
\text { vivienda }\end{array}$ & $\begin{array}{c}\text { Autoper- } \\
\text { cepción }\end{array}$ & $\begin{array}{c}\text { Depen- } \\
\text { dencia }\end{array}$ & $\begin{array}{c}\text { Nivel de } \\
\text { instrucción }\end{array}$ & $\begin{array}{c}\text { Situación } \\
\text { laboral }\end{array}$ & $\begin{array}{c}\text { Condiciones de } \\
\text { hacinamiento }\end{array}$ \\
\hline Estrato 1 & 1 & 1 & 0 & 1 & 1 & 0 \\
(De mayores recursos) & Normal & Media-alta & Normal & Avanzado & Normal & No \\
Estrato 2 & 1 & 0 & 0 & 0 & 0 & 1 \\
(Intermedio) & Normal & Baja & Normal & Bajo & Marginal & Sí \\
Estrato 3 & 0 & 0 & 1 & 1 & 0 & 1 \\
(De menores recursos) & Precaria & Baja & Alta & Bajo & Marginal & Sí \\
\hline
\end{tabular}


FIGURA 1. Representación porcentual de los tres subgrupos socioeconómicos atendidos en cinco hospitales públicos de Lima, Perú, en 1988, 1992 y 1997

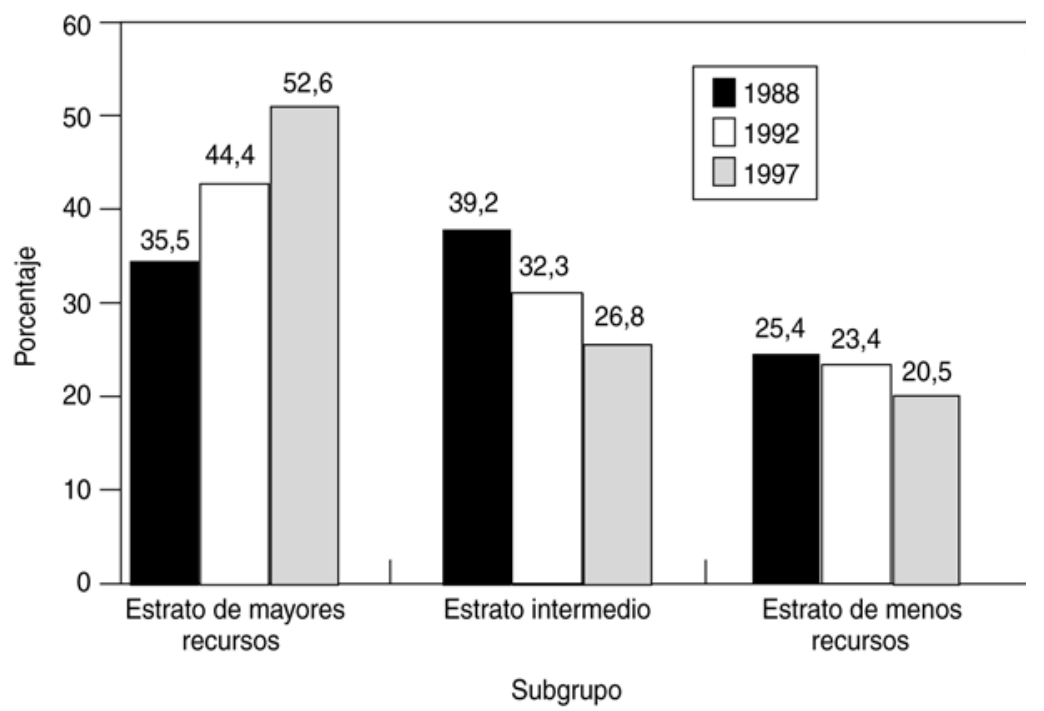

de 52,7 a $40,7 \%$ y los subgrupos 2 y 3 (relativamente más pobres) crecieron de 29,1 a $33,3 \%$ y de 11,1 a $25,9 \%$, respectivamente. Un análisis más detallado muestra que en la demanda atendida de este último hospital no hubo una tendencia uniforme a lo largo del decenio. Los pacientes más pobres disminuyeron entre 1988 y 1992 y luego aumentaron notablemente entre 1992 y 1997. Este fenómeno probablemente pueda explicarse si se tiene en cuenta la incorporación de la población de San Juan de Lurigancho, uno de los distritos más pobres de Lima, al público tradicional del Hospital Hipólito Unanue en el período estudiado.

Cabe notar que factores externos a la dinámica del sector salud y de los hospitales han incidido en el comportamiento de la demanda en salud, lo que se expresa en las variaciones de la tendencia general de disminución de los subgrupos 2 y 3 del público hospitalario. Sin embargo, como se discutirá a renglón seguido, es probable que los factores centrales que pueden explicar lo sucedido hayan estado principalmente relacionados con la orientación de la oferta de atención y no con la evolución de la distribución de ingresos a nivel nacional. La reforma de la oferta de atención modificó su propia demanda.

De las cuatro modificaciones hospitalarias observadas en los establecimientos estudiados, la de mayor importancia para nuestra investigación fue el verificó midiendo el aumento del peso absoluto y relativo de los recursos generados por los propios hospitales. Deflatando las cifras y tomando como base el año 1990, en el período de 1991 a 1995 los recursos propios del Hospital María Auxiliadora aumentaron $141 \%$; los del Cayetano Heredia, 186,3\%; los del Hipólito Unanue, 224,2\%; los del Daniel Alcides Carrión, 261,4\%; y los del Arzoautofinanciamiento progresivo. Este se

Fuente: Oficinas de Contabilidad de los hospitales. bispo Loayza, 553,9\% (cuadro 2 y figura 2). La curva general de esos ingresos propios revela un crecimiento notable hasta 1992, un decrecimiento leve en 1993 y un nuevo ascenso durante 1994 y 1995. La excepción es el hospital María Auxiliadora, el único de los cinco que tuvo un leve descenso de ingresos propios desde 1992 hasta 1995. El hospital cuyos recursos propios aumentaron más fue el Arzobispo Loayza, seguido del Daniel Alcides Carrión. Actualmente, los recursos propios financian buena parte de los bienes y servicios necesarios para la operación de los establecimientos. El aporte del Estado se nota, fundamentalmente, en el rubro de remuneraciones.

Sin embargo, el aumento de los recursos autogenerados por los hospitales no puede atribuirse solamente a los ingresos provenientes de las tarifas por consultas, pues incluye también los ingresos devengados de las farmacias hospitalarias, la venta de servicios como los de las clínicas hospitalarias y los análisis de laboratorio y otros rubros. Es decir, ese incremento de recursos propios no se debe solo a la política de tarifas, sino en general al nuevo modelo hospitalario en construcción, que involucra la diversificación y creación de nuevas fuentes de ingreso y la venta de servicios (13). Los últimos estudios auspiciados por el MINSA muestran que los recursos propios han crecido notablemente en el sector salud. Hacia fines de los años ochenta se calcularon en $7 \%$ de los ingresos totales y en 1996, en $17 \%$ (14).

Por otro lado, los ingresos que los cinco hospitales recibían del tesoro pú-

CUADRO 2. Ingresos anuales autogenerados por 5 hospitales públicos de Lima, Perú, de 1991 a 1995. Cifras deflatadas, año base: 1990. Miles de soles

\begin{tabular}{lrcccr}
\hline & \multicolumn{5}{c}{ Año } \\
\cline { 2 - 6 } \multicolumn{1}{c}{ Hospital } & 1991 & 1992 & 1993 & 1994 & 1995 \\
\hline Hipólito Unanue & 94114 & 190677 & 174147 & 193854 & 229895 \\
Arzobispo Loayza & 103165 & 143701 & 192232 & 479156 & 571490 \\
María Auxiliadora & 159734 & 245865 & 232205 & 227744 & 225265 \\
Cayetano Heredia & 171029 & 175046 & 181609 & 228139 & 318769 \\
Daniel A. Carrión & 143107 & 218403 & 195499 & 305939 & 374120 \\
\hline
\end{tabular}


FIGURA 2. Ingresos anuales autogenerados por cinco hospitales públicos, Lima, Perú, 1991 a 1995

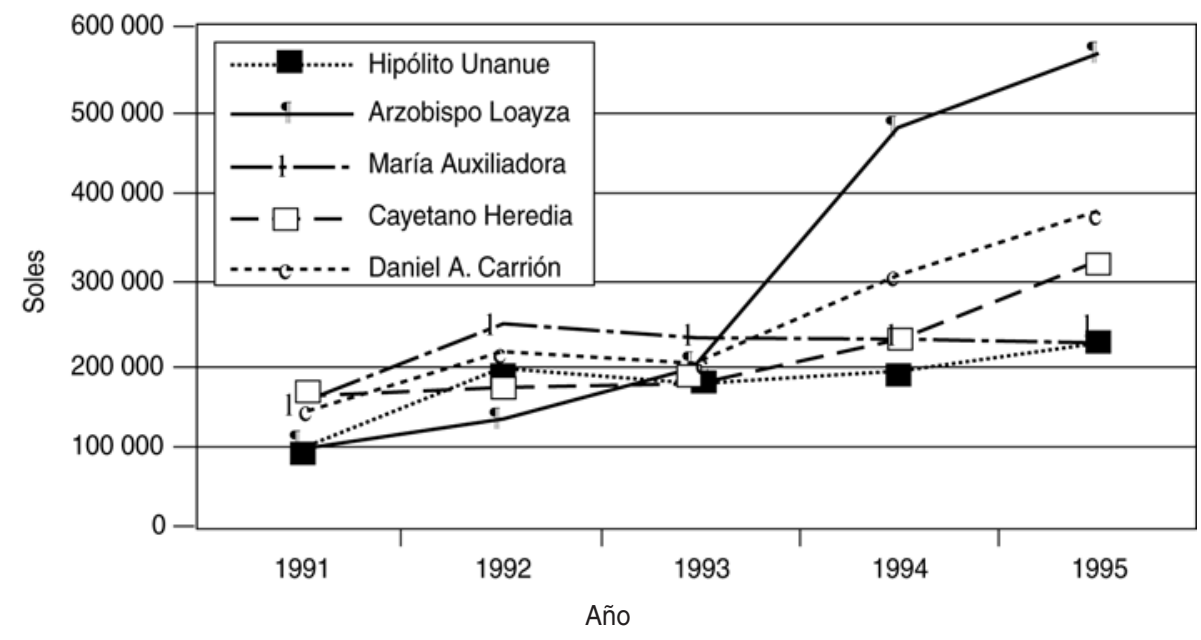

CUADRO 3. Porcentajes anuales de los recursos autogenerados (RA) y los recibidos del tesoro público (TP) por cinco hospitales de Lima, Perú, 1991 a 1995

\begin{tabular}{|c|c|c|c|c|c|c|}
\hline \multirow[b]{2}{*}{ Hospital } & & \multicolumn{5}{|c|}{ Año } \\
\hline & & 1991 & 1992 & 1993 & 1994 & 1995 \\
\hline \multirow[t]{2}{*}{ María Auxiliadora } & $\mathrm{RA}$ & 19,7 & 28,6 & 25,8 & 24,3 & 19,9 \\
\hline & TP & 80,3 & 71,4 & 74,2 & 75,7 & 80,1 \\
\hline \multirow[t]{2}{*}{ Daniel A. Carrión } & $\mathrm{RA}$ & 13,4 & 17,8 & 15,9 & 20,0 & 20,0 \\
\hline & TP & 86,6 & 82,2 & 84,1 & 80,0 & 80,0 \\
\hline \multirow[t]{2}{*}{ Cayetano Heredia } & $\mathrm{RA}$ & 18,5 & 17,9 & 18,0 & 21,4 & 24,3 \\
\hline & TP & 81,5 & 82,1 & 82,0 & 78,6 & 75,7 \\
\hline \multirow[t]{2}{*}{ Arzobispo Loayza } & $\mathrm{RA}$ & 8,6 & 11,5 & 14,6 & 28,0 & 28,1 \\
\hline & $\mathrm{TP}$ & 91,4 & 88,5 & 85,4 & 72,0 & 71,9 \\
\hline \multirow[t]{2}{*}{ Hipólito Unanue } & RA & 11,75 & 21,2 & 19,7 & 19,9 & 19,8 \\
\hline & TP & 88,25 & 78,8 & 80,3 & 80,1 & 80,2 \\
\hline
\end{tabular}

CUADRO 4. Producción anual de consultas externas en cinco hospitales públicos, Lima, Perú, 1991 a 1995

\begin{tabular}{|c|c|c|c|c|c|}
\hline \multirow[b]{2}{*}{ Hospital } & \multicolumn{5}{|c|}{ Número de consultas al año } \\
\hline & 1991 & 1992 & 1993 & 1994 & 1995 \\
\hline Arzobispo Loayza & 99700 & 176200 & 207400 & 226100 & 259200 \\
\hline María Auxiliadora & 85910 & 141541 & 138958 & 206054 & 238860 \\
\hline Cayetano Heredia ${ }^{a}$ & 77385 & 124200 & 133230 & 130768 & 147671 \\
\hline Hipólito Unanue ${ }^{b}$ & 85971 & 136548 & 168845 & 200014 & 214307 \\
\hline Daniel A. Carrión & 75630 & 139230 & 116726 & 120337 & 163330 \\
\hline
\end{tabular}

Fuente: Perú, Ministerio de Salud, Oficina General de Estadística e Informática.

a En 1991 hubo tres meses de huelga.

${ }^{b}$ En 1991 hubo tres meses de huelga. blico no disminuyeron en términos absolutos, aunque sí en términos relativos. En los últimos años el tesoro público ha pesado menos proporcionalmente en los presupuestos hospitalarios. En términos reales, las transferencias del Estado se mantuvieron estacionarias entre 1991 y 1993, para luego comenzar un aumento notable. En 1995, el hospital Daniel Alcides Carrión recibió del tesoro público $44,12 \%$ más que en 1993; el Hipólito Unanue, 30,3\% más; el Arzobispo Loayza, 30,2\% más; el María Auxiliadora, 35,7\% más y el Cayetano Heredia, 19,7\% más. En términos relativos, los hospitales Daniel A. Carrión y María Auxiliadora fueron los que tuvieron un aumento mayor de ingresos del tesoro público con respecto a las partidas que recibían en 1993.

Sin embargo, comparando la evolución de los ingresos reales en recursos propios con los ingresos reales en tesoro público, se demostró una moderada correlación inversa en la que, a más ingresos propios, menos ingresos del tesoro público. De la suma total de ingresos percibidos en 1991 por el Hospital Daniel A. Carrión por recursos autogenerados y transferencias del estado, los recursos propios ascendían a $13,4 \%$ y los del tesoro público, a $86,6 \%$. En 1995, los recursos propios eran de $20 \%$ y los del tesoro público, de $80 \%$. Los otros hospitales tuvieron experiencias similares, como se observa en el cuadro 3. En otras palabras, mientras más eficiente era un hospital en la generación de recursos propios, más probabilidades tenía de que se redujeran las transferencias de fondos del tesoro público, en términos relativos.

En cuanto a la producción de servicios hospitalarios, la revitalización de actividades fue notable en ese período, como indican las estadísticas sobre consultas externas de los cinco establecimientos estudiados. En el cuadro 4 y la figura 3 puede apreciarse que entre 1991 y 1995 el número de consultas externas casi se triplicó en los hospitales Arzobispo Loayza, María Auxiliadora e Hipólito Unanue, mientras que se duplicó en los hospitales Cayetano Heredia y Daniel Alcides Carrión. 
FIGURA 3. Producción de consultas externas en cinco hospitales públicos, Lima, Perú, 1991-1995

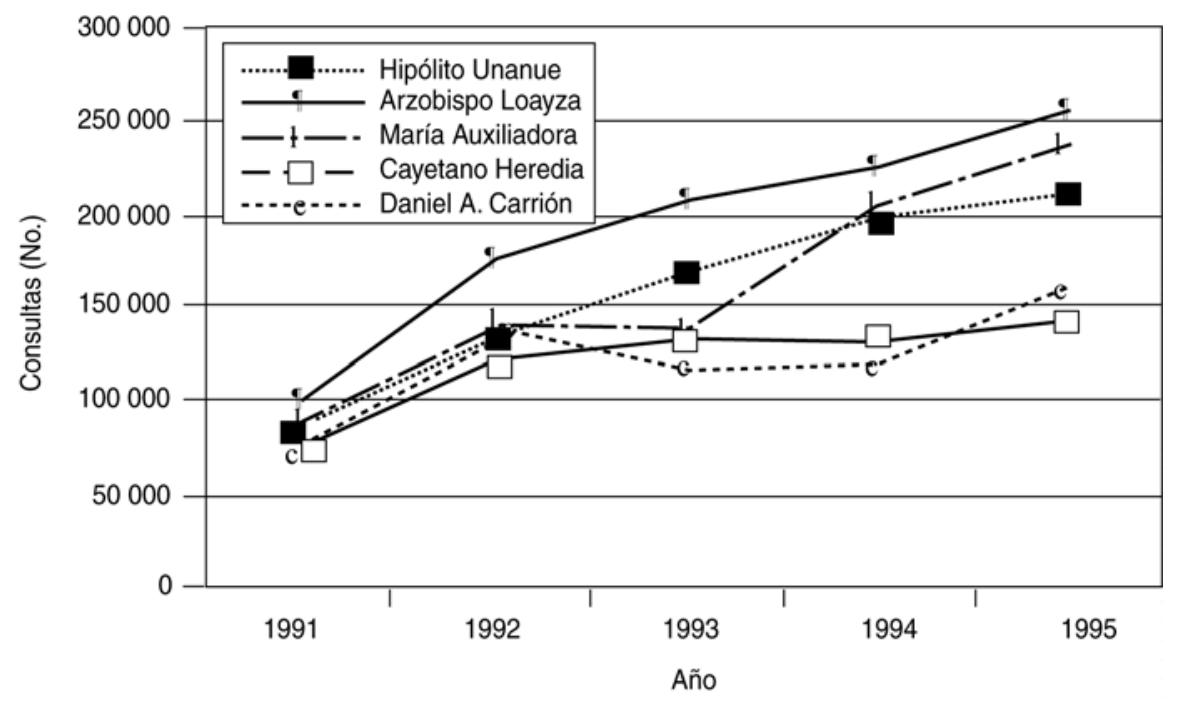

\section{DISCUSIÓN}

Nuestra investigación ratifica diversos estudios nacionales sobre el mismo tema (15-18), en los que se comprobaron la magnitud de la pobreza y la desigualdad social existentes en el Perú $\mathrm{y}$, en algunos de ellos, el sobrepeso de las capas medias en el gasto social público, lo que después fue retomado por la crítica a las políticas sociales basadas en los presupuestos históricos y el subsidio público no orientado. Lo nuevo es que en los años noventa, en un entorno marcado por las ideas de calidad del gasto social y focalización, las políticas sociales todavía no han logrado superar el "subsidio ciego".

Efectivamente, las encuestas nacionales de niveles de vida (ENNIV) de 1991 (19) y 1994 (20) mostraron que de un año al otro la demanda de servicios hospitalarios por parte de las personas extremadamente pobres disminuyó de 30,1 a $28 \%$ y que la de los pobres disminuyó igualmente, de 37,3 a 34\%. Por el contrario, la presencia de personas no pobres en los hospitales aumentó de 34,8 a $43,8 \%$ durante el mismo período. Nuestro estudio ratificó que esa tendencia continuó por lo menos hasta el primer trimestre de 1997, fecha en que se realizó nuestra última encuesta. Las ENNIV también mostraron que, si bien entre 1991 y 1994 los no pobres acudieron con mayor frecuencia a los hospitales, desertaron drásticamente de las clínicas y consultorios al punto de que su presencia en ellos disminuyó de $33,7 \%$ en 1991 a $17,9 \%$ en 1994 . Esos datos nos obligan a concluir que, desde el punto de vista social, la expansión de la productividad hospitalaria que hemos visto desde 1994 se ha dirigido al estrato de pobreza no extrema (personas por debajo de la línea de pobreza sin ser indigentes o pobres extremos); al estrato de pobreza coyuntural, que integra a los "nuevos pobres" provenientes de las capas medias y capas afectadas por la crisis económica; y a estratos de medianos ingresos.

Según los datos hechos disponibles por encuestadoras nacionales, hasta 1997 la dinámica general muestra una mayor presencia de los estratos socioeconómicos pobre y medio en los hospitales públicos y de los pobres, en particular los extremadamente pobres, en los centros y puestos de salud del MINSA. Desde 1994 en adelante, con la reexpansión de los centros y postas llevada a cabo por el Programa Salud Básica para Todos (PSBT) y los Comités
Locales de Administración de Salud (CLAS), ${ }^{2}$ se ha establecido una doble segmentación en el subsector público entre la periferia y los hospitales. En la primera predominan los extremadamente pobres y los pobres; y en los segundos, los pobres y los no pobres.

No es dable presumir que el incremento de usuarios hospitalarios de estratos más pudientes obedezca a una mejora correlativa de los ingresos en el Perú o aun en Lima, pues en todo el período estudiado se ha destacado la magnitud de la pobreza y la indigencia. Entre 1994 y 1996 se registró a nivel nacional un aumento de la pobreza de 46,5 a $49,0 \%$ y en Lima Metropolitana, de 37,2 a 39,7\% (21).

Por otro lado, nuestra investigación se ubica en una línea de investigaciones internacionales que han estado evaluando las consecuencias de la autonomización y el autofinanciamiento de los hospitales públicos en la eficiencia y la equidad. Como han señalado Newbrander, Barnum y Kutzin (22), la preocupación actual por el incremento del gasto en salud se ha concentrado en los hospitales porque estos representan las unidades de mayor costo operativo de los sistemas de salud. En los hospitales de los países en desarrollo se invierte entre 50 y $65 \%$ del gasto público en salud. En el Perú, el porcentaje promedio del gasto corriente en salud que se destina a los hospitales a nivel nacional es de $54 \%$ y, a nivel central, en Lima, de 70\% (14). De ahí la búsqueda de nuevas fuentes de recursos así como de formas más eficientes de gerenciar las operaciones hospitalarias. Sin embargo, como añaden los mismos autores, las políticas de recuperación de costos han originado preocupación en cuanto a sus implicaciones sobre la equidad en el acceso y la utilización de los servicios hospitalarios. Por eso, si bien los primeros estu-

\footnotetext{
2 El PBST y los CLAS son programas para centros y postas periféricas del subsector público creados en 1994 por el gobierno, el primero para repotenciar los servicios básicos en zonas deprimidas y, el segundo, para implementar una modalidad de gestión participativa de los establecimientos periféricos.
} 
dios tendieron a mostrar que la demanda no tenía que ver con los precios de la atención (23), las investigaciones posteriores han marchado en sentido contrario.

Uno de los primeros estudios que llamó la atención a los posibles efectos de la recuperación de costos sobre la demanda hospitalaria en el Perú fue precisamente el que realizaron Gertley et al. dentro de los estudios de Análisis del Sector Salud-Perú, realizados en 1985 y 1986. Los autores establecieron el efecto previsible del establecimiento de tarifas sobre diversos tipos de usuarios mediante un modelo de planificación y simulación (24). Luego Gertley y Van der Gaag volvieron años más tarde al mismo tema (25). Entre tanto, Frankish demostró que el incremento de los precios había reducido el número de consultas externas en una muestra de pacientes con enfermedades crónicas (26). Weaver también concluyó, a partir de un estudio de los pacientes del primer hospital de referencia de Nigeria, que el establecimiento de tarifas modificaba el comportamiento de los pacientes ambulatorios, demorando la decisión de buscar atención médica (27). Por último, cabe mencionar que en 1994 Espinoza y Sanes mostraron que el aumento de los recursos propios del Hospital María Auxiliadora de Lima había generado una disminución de la demanda de servicios de consulta externa (28).

Por la complejidad del problema, en este estudio se ha evitado un planteo reduccionista de causalidad directa entre el establecimiento de tarifas y el número de consultas o el nivel social de los atendidos y se ha intentado más bien revelar los resultados de la reforma tal como se han manifestado en el cambio de usuarios de los hospitales. La calidad de la reforma hospitalaria y sectorial se aborda, no desde el análisis de los modelos de reforma en sí mismos sino desde fuera, en sus resultados. Podría contribuir, por lo tanto, a modificar la tendencia espontánea a concebir la reforma sanitaria como solo reforma de los servicios.
Esta reducción de la reforma incentiva a los directivos de las instituciones de salud a advertir que su gestión va a ser evaluada si sus servicios se vuelven más eficientes, soslayándose la dimensión sectorial, sanitaria y poblacional de la reforma. Felizmente, si la reforma se concibe como un proceso, es decir, no solo como un modelo ni un simple acto gubernativo, sino como una construcción social, cultural y política, es, por lo tanto, perfectible.

De nuestro estudio se desprende que la reforma hospitalaria silenciosa hasta 1997 fue de carácter institucional y no sistémico, y optimizó microeconómicamente los establecimientos de servicios. Sin embargo, podría afectar negativamente a los niveles de equidad del sistema, por lo menos en cuanto al impacto en la población que depende de los hospitales estudiados. Sin embargo, no toda mejora en la productividad y eficiencia de los establecimientos tiene necesariamente que redundar en efectos negativos para la población en términos de acceso y equidad. Hay múltiples maneras de articular eficiencia y equidad, pero el punto de partida es hacer que la eficiencia deje de ser un fin y se convierta en un medio, y la línea de fuerza real ponga de relieve tanto los indicadores de producción y rendimiento como los de impacto $\mathrm{y}$ efectividad.

Siguiendo la orientación de transformar los hospitales públicos en unidades autosustentables, como las denominadas "unidades estratégicas de negocios", durante los años estudiados todos los establecimientos hicieron hincapié en incorporar nuevos enfoques y herramientas de gestión modernas e introducir lo que Humberto Novaes ha denominado "ingeniería gerencial hospitalaria" (29). Pero la ausencia de la dimensión macrosistémica de la reforma - que compete al MINSA - entraña el riesgo de originar un cuadro de microeficiencia con macroineficiencias y macroinequidades. Los hospitales públicos pueden así mejorar su situación, pero no necesariamente la salud de la población menos pudiente. El problema no es solo ni principalmente de los hospitales; la atención de la salud es una responsabilidad sectorial.

En el esquema general de la reforma peruana, se trata de articular elementos del modelo de atención gerenciada con el modelo de contrato público inglés, la reforma gerencial micro con la construcción de cuasimercados. En dicho diseño el hospital será autónomo y el estado le comprará servicios para los sectores pobre e indigente. El problema de la transición es que un despliegue inarmónico de la reforma puede cuajar en modelos no deseados. Como señala acertadamente Maynard (30), la lección derivada de las dificultades de la reforma del Servicio Nacional de Salud inglés es que cualquier paquete de reformas debe ser completo y comprender líneas de reforma que se refuerzan mutuamente. Si un planteo macrosistémico de reforma es débil, ello empuja a los establecimientos a una planificación "hacia dentro" en que se busca aumentar la productividad y los ingresos por tarifas pero sin ubicar los planes en el marco de las necesidades de la población. Ello se debe a la ausencia de un plan mayor y de un diagnóstico social de la demanda de cada hospital, el cual debería existir a partir de la calificación, no solo de los pacientes que solicitan prestaciones gratuitas, sino en general de todos los pacientes. Es evidente, entonces, que se necesita no solo un proyecto técnico-político de reforma global, sino un sistema de evaluación y monitoreo del sentido real de las transformaciones en curso en el sector de la salud en términos de su equidad, eficiencia y calidad.

Agradecimiento. El autor agradece a la Organización Panamericana de la Salud el apoyo financiero que permitió realizar la investigación aquí presentada. Agradece asimismo el apoyo brindado por los doctores Carlos Vidal Layseca y Pedro Brito, y por sus cole- 
gas de la Facultad de Salud Pública, los doctores Alejandro Llanos, Marcos Cueto, Oswaldo Lazo, Luis Lozano, Humberto Castillo, Victor Carrasco y
Eduardo Vidal. Da las gracias del mismo modo, por diversos aportes a la investigación, al Dr. Diego Gonzales, Dr. Fernando Salazar, Mag. Leopoldo
Bejarano, Dr. Francisco Leyva, Lic. Claudia Pacheco, Dr. Luis Chunga, Lic. Patricia Asenjo, Lic. Fernando Bravo y Dra. Marisol Vicuña.

\section{REFERENCIAS}

1. Perú, Ministerio de Salud. Por un sistema nacional regionalizado integrado de salud. Lima: MINSA; 1991.

2. Govindaraj R, Chawla M. Experiencias recientes de autonomía en hospitales de países en desarrollo - ¿Qué podemos aprender? Boston: Harvard School of Public Health; septiembre de 1996. [Este estudio auspiciado por USAID está disponible en español en la página web del Proyecto Datos para la Toma de Decisiones de la Facultad de Salud Pública de la Universidad de Harvard, proyecto dirigido por Peter Bermann.]

3. Glennerster H, Le Grand J. The development of quasi-markets in welfare provision in the United Kingdom. Int J Health Serv 1995;25(2): 203-218.

4. Gutiérrez C, Molina C, Wullner A. Las formas de contratación entre prestadoras y administradoras de salud. Bogotá: Fundación Social, Fundación Corona, FES y Fundación Restrepo Barco; 1995.

5. Eichler R. Mecanismos para el pago de proveedores y de la atención médica administrada: implicaciones para la reforma del sistema de seguridad social. En: Organización Panamericana de la Salud. Conferencia Regional sobre Mecanismos para el Pago de Proveedores, Lima, 16 a 17 de noviembre de 1998.

6. Perú, Ministerio de Salud, Oficina de Estadística e Informática y Programa de Fortalecimiento de Servicios de Salud. II censo de infraestructura y recursos humanos. Lima: MINSA; 1997.

7. Hair J, Anderson R. Multivariate data analysis with reading. 3a. ed. New York: McMillan Publishing Co., 1992.

8. Perú, Instituto Nacional de Estadística e Informática. Metodología para determinar el in- greso y la proporción de hogares pobres. Lima: INEI, 1996.

9. Cuanto S.A. Encuestas nacionales de niveles de vida 1991, 1994 y 1996. Lima: Cuanto S.A.

10. Apoyo Opinión y Mercado S.A. Informe gerencial de marketing. Niveles socio-económicos. Lima: Apoyo; 1997.

11. Paganini JM, Novaes H. El hospital público, tendencias y perspectivas. Washington, D.C: Organización Panamericana de la Salud; 1994. (HSS/SILOS-39).

12. Perú, Ministerio de Salud. Lineamientos de políticas de salud, 1995-2000. Lima: MINSA; 1995.

13. Joo C, Juárez E, Sara J, Lama A, Orcasitas L. Informe de gestión del Hospital Carrión, 1990-1995. Callao: trabajo inédito; 1996.

14. Macroconsult S.A. Análisis del gasto público en salud. Lima: Ministerio de Salud [Proyecto 2000]; 1997.

15. Webb R. The distribution of income in Peru. Princeton University, Research Program in Economic Development; 1972. (Discussion paper 26).

16. Webb R, Figueroa A. Distribución del ingreso en el Perú. Lima: Instituto de Estudios Peruanos; 1975.

17. Amat y León C. Distribución del ingreso familiar en el Perú. Lima: Centro de Investigaciones de la Universidad del Pacífico; 1979.

18. Habich M. Un análisis exploratorio de la distribución del ingreso en el Perú. Lima: Banco Central de Reserva; 989.

19. Cuanto S.A. Niveles de vida - Perú: subidas y caídas, 1991. Lima: Cuanto; 1993.

20. Cuanto S.A. Retrato de la familia peruana: niveles vida, 1994. Lima: Cuanto; 1995.

21. Cuanto S.A. Mil quinientas familias dos años después: la pobreza en el Perú, 1994-1996. Lima: Cuanto; 1996.
22. Newbrander W, Barnun H, Kutzin J. Hospital economics and financing in developing countries. Geneva: World Health Organization; 1992. (SHS/NHP/92.2).

23. Akin J, Guilkey D, Popkin P. The demand for child health services in the Philippines. Soc Sci Med 1981;15:249-257.

24. Gertley P, Locay L, Peñaranda C, Sanderson W, Torres W. Demanda de servicios de salud en el Perú. Lima: Análisis del Sector SaludPerú; 1986.

25. Gertley P, van der Gaag J. The willingness to pay for medical care: evidence from two developing countries. Johns Hopkins University Press; 1990.

26. Frankish J. Day hospital fees and accessibility of essential health services. South Afr Med J 1986;70:285-288.

27. Weaver M. User fees and patient behaviour: evidence from Niamey National Hospital. Health Policy Plann 1995;10(4):350-361.

28. Espinoza J, Sanes E. Impacto del incremento de los costos de atención sore la demanda de los servicios. Rev Per Epidemiol 1994;7:16-19.

29. Novaes H. Calidad total: nuevo recurso para los hospitales de América Latina. El ingeniero gerencial. Educ Med Salud 1994;28:490-506.

30. Maynard A. Can competition enhance efficiency in health care? Lessons from the reform of the U. K. National Health Service. Soc Sci Med 1994;39:1433-1445.

Manuscrito recibido el 6 de febrero de 1998 y aceptado para publicación, tras revisión, el 29 de junio de 1999 
ABSTRACT Between 1988 and 1992 public hospitals in Peru were affected by major changes: the operational collapse of the health services, a profound State budgetary crisis, and a series of adjustments aimed toward self-financing. These changes all came before the

\section{Greater autonomy for public} hospitals in Lima and the consequences of that on the use of health care services, 1988-1997 health sector reform laws of 1997. In recent years, the changes in financing policy have renewed the operational potential of hospital activity and of the entire health sector. On the other hand, there have been some signs that with hospitals generating their own resources through fee collection, there has been a shift in the proportion of hospital users from different socioeconomic strata, to the detriment of poorer persons, especially those who need free services. This study tried to determine if there had been such changes between 1988 and 1997 in the socioeconomic strata of persons using five large public hospitals in Lima, and the association that that could have with the current transition toward the new model of the autonomous hospital, particularly with the new policies on hospital financing.

Changes in hospital usage were studied through in-depth interviews with key informants, collection of statistical data in the selected hospitals, and an extensive bibliographic and literature review. Changes in hospital usage were studied through 1012 surveys done with hospital users from the years 1988, 1992, and 1997. In order to classify those persons by socioeconomic stratum, cluster analysis and discriminant analysis were used, based on six variables suitable for determining socioeconomic level. The resulting model, of three strata grouped according to the dichotomized variables, exceeded the 75-point minimum required for the discriminant analysis to confirm the classification test.

The main results showed that among the users of the five Lima hospitals, those in the highest stratum increased their relative use of the hospitals from $35.4 \%$ in 1988 to $52.6 \%$ in 1997. Proportional usage by those in the lowest stratum declined in the same period, from $25.4 \%$ to $20.5 \%$. Usage by those in the intermediate stratum also declined, from $39.2 \%$ to $26.8 \%$. It is clear that the trend in hospital financing has been toward an increase in user copayments, with public funding making up a smaller proportion of hospital budgets. This study verifies the results found in the 1991 and 1994 National Surveys of Standards of Living, which showed that the proportion of hospital usage by "poor" persons declined from 37.3\% to 34\% between 1991 and 1994 and that usage by persons above that "poor" economic level increased from $34.8 \%$ to $43.8 \%$. The authors conclude that without a holistic approach to reform, microreforms produce both microefficiency and systemic inequity. In those circumstances, output and yield become more important than the impact and effectiveness of services for the population served. 